\title{
Localized Pigmented Villonodular Synovitis of the Proximal Tibiofibular Joint
}

\author{
Jae Ho Kwon, $\mathrm{MD}^{1}$, Jae Hwi Han, $\mathrm{MD}^{1}$, Vivian RD’ Almeida, $\mathrm{MS}^{1}$, Seong Hyun Kim, $\mathrm{MD}^{2}$, Hai Jin Park, $\mathrm{MD}^{2}$, \\ and Kyung-Wook Nha, $\mathrm{MD}^{1}$ \\ Departments of ${ }^{1}$ Orthopaedic Surgery and ${ }^{2}$ Dermatology, Inje University Ilsan Paik Hospital, Ilsan, Korea
}

\begin{abstract}
Pigmented villonodular synovitis (PVNS) is a rare disease. It is a benign neoplastic process typically affecting young to middle-aged adults and most commonly involving the knee, hip, and shoulder joints. The symptoms include diffuse pain and swelling with discomfort. We report a rare case of localized PVNS originating at the proximal tibiofibular joint in a 39-year-old female patient with radiologic changes for short duration of time. The clinical history, plain radiographs, magnetic resonance imaging, and pathologic findings of the reported patient were reviewed. Complete surgical excision was performed and there was no evidence of recurrence after one-year follow-up.
\end{abstract}

Keywords: Pigmented villonodular synovitis, Tibiofibular joint

Pigmented villonodular synovitis (PVNS) is a rare, benign proliferating disorder of the synovium which can be locally aggressive affecting the joints, tendon sheaths and bursa. It is more commonly seen in young and middle-aged adults ${ }^{1)}$. It is often monoarticular with the knee being the most common joint to be involved $^{1,2)}$. The other common sites of occurrence include the hip joints, the flexor tendon sheaths of the hand, the ankle and shoulder joints ${ }^{3)}$. The sternoclavicular and tibiofibular joints are rare locations ${ }^{2}$. Patients frequently present with pain, joint effusions and swelling. The disease can present in the knee joint in two forms, diffuse and localized ${ }^{1,2)}$. Plain radiography may show erosions in the adjacent bone. Magnetic resonance imaging (MRI) is useful to delineate the soft tissue extent, evaluate the bony involvement and the typical signal intensity changes seen on T1-

Received December 9, 2013; Revised January 28, 2014;

Accepted February 21, 2014

Correspondence to: Kyung-Wook Nha, MD

Department of Orthopaedic Surgery, Inje University Ilsan Paik Hospital, 170 Juhwa-ro, Ilsanseo-gu, Goyang 411-706, Korea

Tel: +82-31-910-7312, Fax: +82-31-910-7319

E-mail: kwnhamj@hotmail.com

This is an Open Access article distributed under the terms of the Creative Commons Attribution Non-Commercial License (http://creativecommons.org/licenses/by-nc/3.0/) which permits unrestricted non-commercial use, distribution, and reproduction in any medium, provided the original work is properly cited. weighted and T2-weighted images ${ }^{4}$. We present a report of localized PVNS arising from both anterior and posterior aspects of the proximal tibiofibular joint with bony erosion at the tip of the fibular in short periods.

\section{Case Report}

A 39-year-old female presented with a three month history of right knee pain and swelling. There was no history of trauma. The pain was diffuse and dull in character. Physical examination revealed an ill-defined swelling at the proximal tibiofibular joint area. Tenderness was diffuse and mild in nature with a rubbery hard consistency and the mass was not mobile in any plane. There was no neurological symptom or sign.

A private clinic made a provisional diagnosis of a ganglion or a lipoma and decided to do an excision biopsy under local anaesthesia. However, it was discovered during surgery that the soft tissue mass was arising from the proximal tibiofibular joint and communicating with the anterior and posterior aspects of the joint. The partially resected specimen was sent for histopathological examination and the results revealed PVNS. The patient was referred to this hospital for further evaluation and treatment.

Plain radiography showed a small sized bony erosion of the fibular tip (Fig. 1). MRI showed a well circumscribed lesion with a capsular margin in the posterior aspect of the proximal tibiofib- 
ular joint with decreased heterogeneous signal intensity on both T1-weighted and T2-weighted images. The T2-weighted image showed a relatively hyper-intense signal compared to the skeletal muscle (Fig. 2A and 2B).

Complete excision and biopsy was done through a posterolateral incision (Fig. 3). Intraoperatively, the mass was yellowish brown in colour, arising from the proximal tibiofibular joint and circumscribing the joint. The mass was carefully isolated and excised totally. Pathological findings of the biopsy specimens showed proliferation of round to polygonal cells, with scant cytoplasm forming villous and fingerlike or rounded mass underlying the synovium. Multinucleated giant cells were scattered throughout the lesion. Hemosiderin-laden macrophages varied

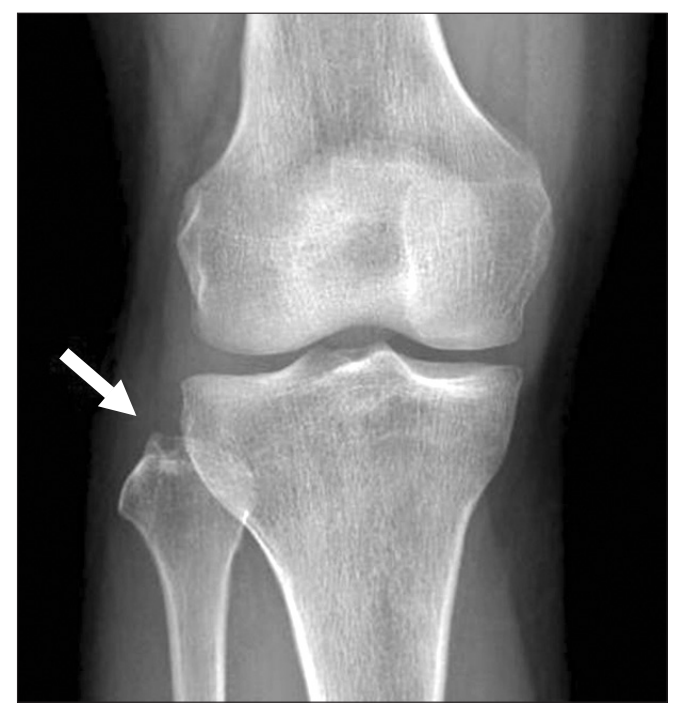

Fig. 1. Plain radiograph showing bony erosion at the tip of the fibular head (arrow). in number, giving the synovium a dark-brown appearance. These findings findings confirmed the diagnosis of localized PVNS (Fig. 4). Postoperative recovery was uneventful and at the most recent follow-up at 12 months after surgery, the patient was fully asymptomatic with no evidence of recurrence on MRI.

\section{Discussion}

Although PVNS is a benign disease, it can be locally destructive causing significant and permanent damage to the joint. The distribution of lesion location depends on the lesion subtypes, either localized or diffuse, and on whether the lesion is intra-articular or extra-articular ${ }^{3,5)}$. Granowitz et al. ${ }^{6}$ classified PVNS into diffuse

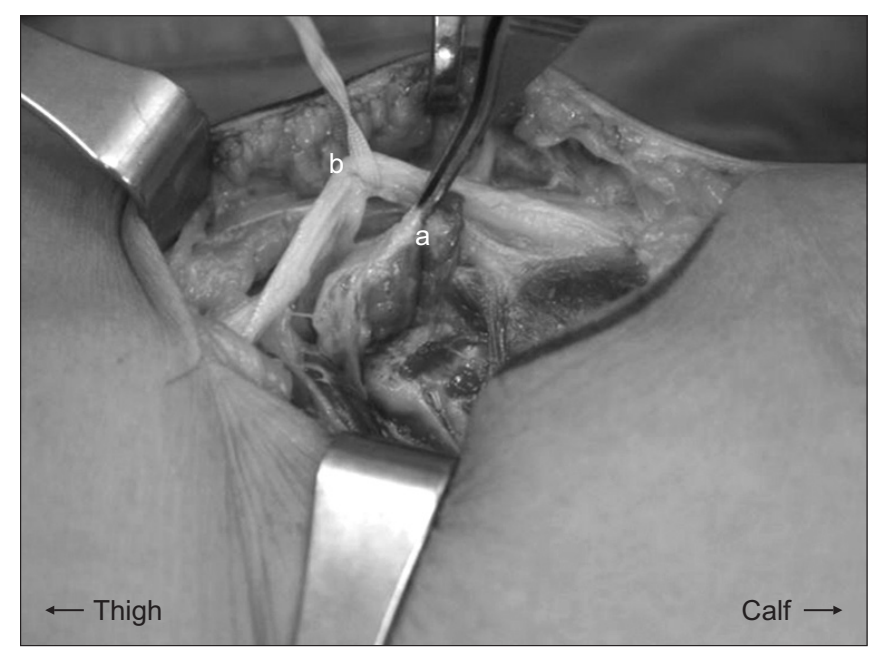

Fig. 3. Intraoperative appearance of the mass excision with a posterolateral approach. a: pigmented villonodular synovitis lesion, b: common peroneal nerve.
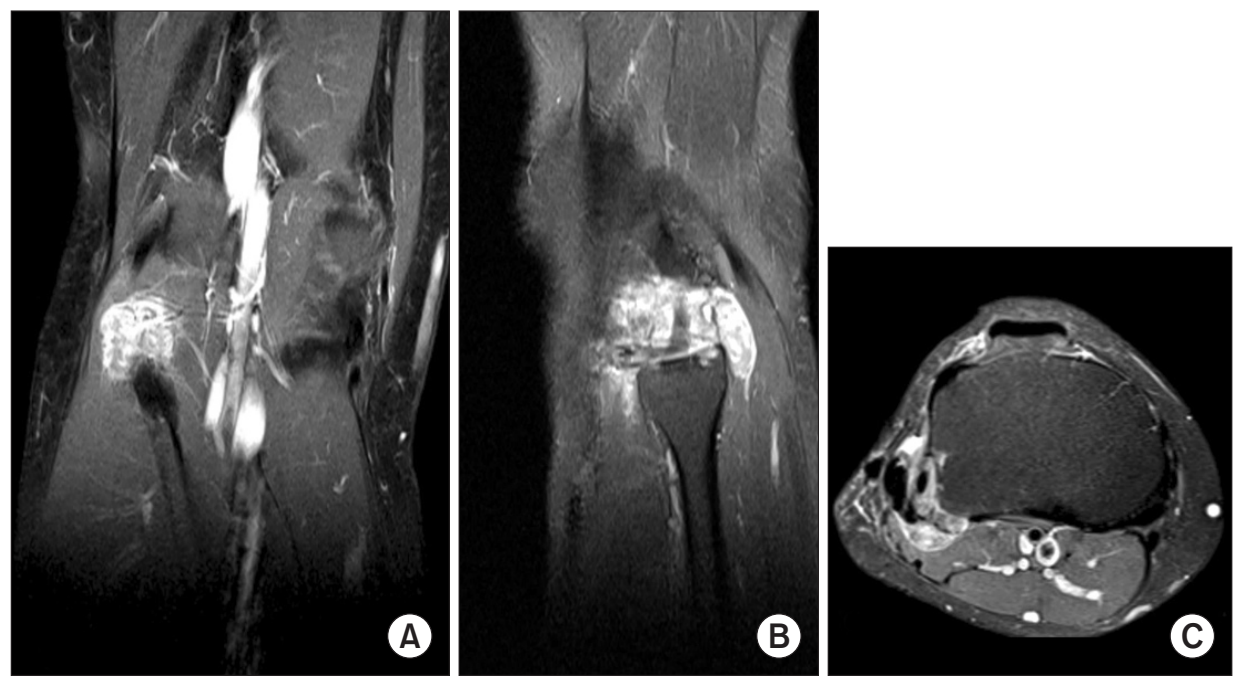

Fig. 2. Enhanced coronal (A), sagittal (B) and axial $(C)$ magnetic resonance images of the right knee showing a well circumscribed lesion with a capsular margin in the posterior aspect of the proximal tibiofibular joint with increased heterogeneous signal intensity. 


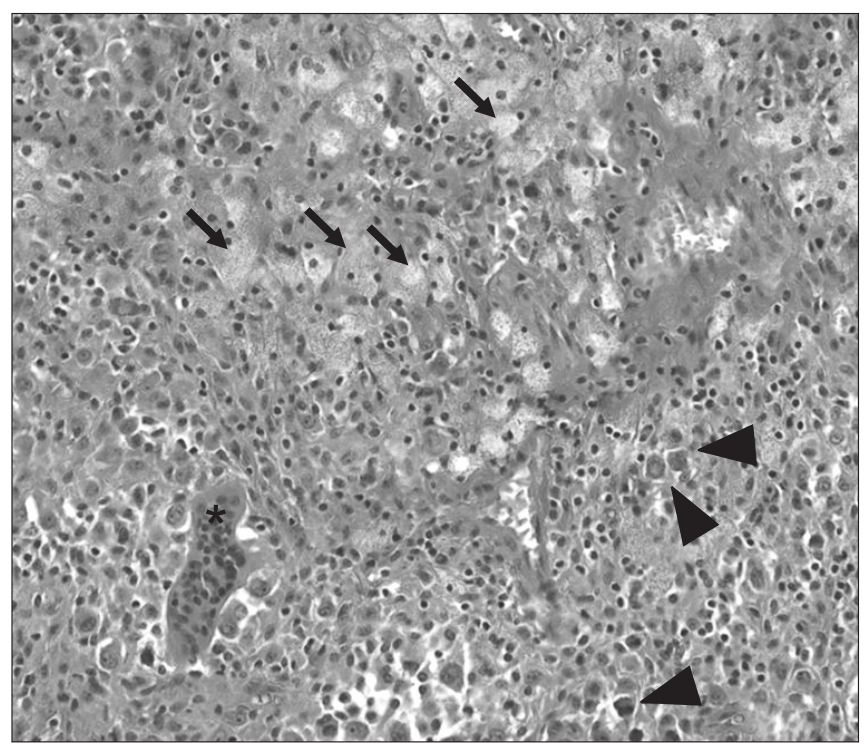

Fig. 4. Histopathological analysis of the biopsy specimen showing the typical appearance of pigmented villonodular synovitis with foam cells (arrows), hemosiderin-containing macrophages (arrowheads), and multinucleated giant cells (asterisk) $(\mathrm{H} \& \mathrm{E}, \times 200)$.

and localized types. Localized PVNS is an uncommon pathology characterized by the presence of nodular pedunculated mass protruding into the joint cavity ${ }^{7}$. Extra-articular lesions are rare and tend to occur in the same joint locations as intra-articular lesions $^{8)}$.

This case report presents a rare site of occurrence of which only one citation is found in the English-language literature. Ryan et al. ${ }^{2)}$ reported a case of PVNS at the proximal tibiofibular joint which was located just anterior to the tibiofibular joint. However, in our case, the mass was arising from the proximal tibiofibular joint extending to the extra-articular lesion and communicated with the anterior and posterior aspects of the joint.

The diagnosis of localized PVNS is difficult, and the time period between the onset of symptoms and the definitive diagnosis is usually two years ${ }^{4)}$.

Plain radiographs are usually unremarkable. However, in about $9 \%$ to $25 \%$ of the total cases, extrinsic erosion, often with well-defined sclerotic margins of the underlying bone may be seen as in our case where the simple radiograph showed bony erosion at the tip of the fibular head. MRI is the method of choice for diagnosis and follow-up. It helps to delineate the location, size, and extent of the lesion ${ }^{4,9)}$. The lesion usually has heterogeneous signal intensity depending on the amount of hemosiderin content and is characterized by decreased intensity on both the T1-weighted and T2-weighted images ${ }^{7,10)}$. Albeit sensitive, MRI is not specific due to other conditions such as fibroxanthoma, hemangioma, and desmoid tumors giving similar pictures ${ }^{7}$.

Histologically, the lesion is characterized by proliferation of polygonal mononuclear cells, histiocytes, scattered multinucleated giant cells, and foam cells with hemosiderin pigment deposits ${ }^{10)}$. The recurrence rate of localized PVNS has been reported to range from $0 \%$ to $13 \%{ }^{4,10)}$, whereas the recurrence rate of diffuse PVNS has been reported to range from $8 \%$ to $35 \%{ }^{7}$.

The location of PVNS described in this article, which originated at the proximal tibiofibular joint, is extremely rare. PVNS that involves small joints such as the tibiofibular joint can be easily misdiagnosed as other mass lesions. Therefore, the clinicians should pay more attention and must consider different diagnosis for mass lesions around the knee joint. Complete surgical excision is the treatment of choice and the patient should be followed up for recurrence.

\section{Conflict of Interest}

No potential conflict of interest relevant to this article was reported.

\section{Acknowledgments}

This article is supported by a 2015 Inje University Research Grant.

\section{References}

1. Tyler WK, Vidal AF, Williams RJ, Healey JH. Pigmented villonodular synovitis. J Am Acad Orthop Surg. 2006;14:37685.

2. Ryan RS, Louis L, O'Connell JX, Munk PL. Pigmented villonodular synovitis of the proximal tibiofibular joint. Australas Radiol. 2004;48:520-2.

3. Yamashita H, Endo K, Enokida M, Teshima R. Multifocal localized pigmented villonodular synovitis arising separately from intra- and extra-articular knee joint: case report and literature review. Eur J Orthop Surg Traumatol. 2013;23 Suppl 2:S273-7.

4. Mandelbaum BR, Grant TT, Hartzman S, Reicher MA, Flannigan B, Bassett LW, Mirra J, Finerman GA. The use of MRI to assist in diagnosis of pigmented villonodular synovitis of the knee joint. Clin Orthop Relat Res. 1988;(231):135-9.

5. Jobe CM, Raza A, Zuckerman L. Pigmented villonodular synovitis: extrasynovial recurrence. Arthroscopy. 2011;27: 1449-51. 
6. Granowitz SP, D'Antonio J, Mankin HL. The pathogenesis and long-term end results of pigmented villonodular synovitis. Clin Orthop Relat Res. 1976;(114):335-51.

7. Kim RS, Lee JY, Lee KY. Localized pigmented villonodular synovitis attached to the posterior cruciate ligament of the knee. Arthroscopy. 2003;19:E32-5.

8. Wagner ML, Spjut HJ, Dutton RV, Glassman AL, Askew JB. Polyarticular pigmented villonodular synovitis. AJR Am J
Roentgenol. 1981;136:821-3.

9. De Ponti A, Sansone V, Malchere M. Result of arthroscopic treatment of pigmented villonodular synovitis of the knee. Arthroscopy. 2003;19:602-7.

10. Perka C, Labs K, Zippel H, Buttgereit F. Localized pigmented villonodular synovitis of the knee joint: neoplasm or reactive granuloma? A review of 18 cases. Rheumatology (Oxford). 2000;39:172-8. 